\title{
A Strategy Using Photodynamic Therapy and Clofibric Acid to Treat Peritoneal Dissemination of Ovarian Cancer
}

\author{
Yoshihito Yokoyama*, Tatsuhiko Shigeto, Rie Miura, Asami Kobayashi, Makito \\ Mizunuma, Aisa Yamauchi, Masayuki Futagami, Hideki Mizunuma
}

\begin{abstract}
Background: The current study examined the effectiveness of concurrent therapy using photodynamic therapy (PDT) and clofibric acid (CA) to treat peritoneal carcinomatosis resulting from ovarian cancer. Materials and Methods: Nude rats were used to create a model of peritoneal carcinomatosis resulting from ovarian cancer and the effectiveness of PDT with 5-aminolevulinic acid methyl ester hydrochloride (methyl-ALA-PDT) was determined. The survival time of rats receiving that therapy was compared to the survival time of a control group. Rats with peritoneal carcinomatosis resulting from ovarian cancer were divided into 3 groups: a group that received debulking surgery (DS) alone, a group that received DS+methyl-ALA-PDT, and a group that received DS+methyl-ALA-PDT+CA. The survival time of the 3 groups was compared. Protoporphyrin, a metabolite of methyl-ALA, produces a photochemical action when activated by light. The level of protoporphyrin (the concentration) that reached organs in the abdomen was measured with HPLC. Results: Rats receiving methylALA-PDT had a significantly longer survival time compared to the controls. Rats with peritoneal carcinomatosis that received DS+methyl-ALA-PDT+CA had a significantly longer survival time compared to the rats that received DS alone. Some of the rats that received concurrent therapy survived for a prolonged period. Protoporphyrin was highly concentrated in peritoneal metastases, but only small amounts reached major organs in the abdomen. PDT was not found to result in necrosis in the intestines. Conclusions: The results indicated that concurrent therapy consisting of PDT with methyl-ALA and CA is effective at treating peritoneal carcinomatosis resulting from ovarian cancer without damaging organs.
\end{abstract}

Keywords: Photodynamic therapy - clofibric acid - peritoneal carcinomatosis - ovarian cancer - 5-aminolevulinic

Asian Pac J Cancer Prev, 17 (2), 775-779

\section{Introduction}

Combinations of taxanes and platinum compounds have resulted in advanced ovarian cancer having a response rate of 70-80\% (Akeson et al., 2008). However, $70 \%$ of ovarian cancers recur, so advanced ovarian cancer ultimately has a 5-year survival rate of $30-40 \%$ (Akeson et al., 2008). Ovarian cancer has the highest mortality rate among gynecologic malignancies (Siegel et al., 2013). One characteristic of the recurrence of ovarian cancer is peritoneal metastasis. Ascites pools, causing abdominal distention and ileus, and this markedly impacts patient QOL. Chemotherapy and secondary debulking surgery have demonstrated effectiveness at treating the recurrence of ovarian cancer (Lee et al., 2015), but surgery to treat peritoneal dissemination is often infective and those metastases display resistance to anticancer drugs. Concerns about treatment are a frequent occurrence. Thus, molecularly targeted drugs are being developed to treat recurrent ovarian cancer, but none is a magic bullet (Kroep et al., 2012; Yu et al., 2014).
Clofibric acid (CA) is a peroxisome proliferatoractivated receptor $\alpha$ ligand. The current authors previously used CA to treat experimentally induced ovarian cancer in mice (Yokoyama et al., 2007). CA has been found to display antitumor action equivalent to that of cisplatin, which is a key drug used to treat ovarian cancer (Yokoyama et al., 2007). CA's antitumor action is related to the inhibition of angiogenesis and the induction of apoptosis (Yokoyama et al., 2007). CA is a major component of the fibrate drugs that are used to treat hyperlipidemia, and CA is a drug that is widely used in routine practice.

Photodynamic therapy (PDT) is a therapy that uses photosensitizers (or their precursors) with an affinity for cancer and visible light to trigger a photochemical reaction. Reactive oxygen species are generated in cancer tissue, causing the tissue to die. One feature of PDT is that a lesion that is apparent endoscopically can be irradiated with a laser beam. PDT has already been used in gynecology to treat early cancer of the cervix (Choi et al., 2014). Moreover, PDT is also used to treat superficial esophageal cancer, early lung cancer, and 
early gastric cancer (Casson,2009; Ikeda et al., 2011; Spinelli et al., 1992). Intra-abdominal metastases (and more specifically peritoneal dissemination) are apparent laparoscopically and can readily be irradiated with a laser beam, so these metastases could be target lesions for PDT. Five-aminolevulinic acid methyl ester hydrochloride (methyl-ALA) is a photosensitizer precursor with an affinity for cancer. When $250 \mathrm{mg} / \mathrm{kg}$ of methyl-ALA was intraperitoneally administered to colon cancer-bearing mice and the lesions were irradiated with light, the tumor shrank (Peng et al., 2001). The current authors have used an ovarian cancer cell line to produce subcutaneous tumors in nude mice (Wakui et al., 2010). When these mice received PDT with methyl-ALA, significant tumor shrinkage resulted (Wakui et al., 2010) PDT with methylALA has antitumor action that is related to the inhibition of angiogenesis and the induction of apoptosis (Wakui et al., 2010).

The basic procedure for surgical treatment of advanced ovarian cancer is debulking surgery (DS). In an experiment with rats, the current authors prolonged the survival time of rats with peritoneal carcinomatosis merely by resection the omentum where disseminated lesions had formed (Yokoyama et al., 2012). Nonetheless, DS alone was inadequate since peritoneal metastases spread throughout the abdominal cavity and ultimately resulted in death (Yokoyama et al., 2012). The current study has sought to determine whether or not PDT is effective in treating peritoneal carcinomatosis resulting from ovarian cancer. This study has also examined the effect that concurrent treatment with PDT and CA has on peritoneal dissemination of ovarian cancer after DS. The extent to which photosensitizers (or their precursors) with an affinity for cancer are taken up by peritoneal metastases is unclear. Such a therapy causes necrosis of peritoneal lesions, but its effects on organs in the abdomen, like the intestines, are also unclear. This study has also examined these issues that arise when using PDT.

\section{Materials and Methods}

\section{Cell line and cell culture}

DISS was kindly provided from Dr. Saga (Jichi Medical School, Tochigi, Japan) (Yokoyama et al., 2007). This cell line was derived from human epithelial ovarian adenocarcinoma, grown in RPMI 1640 medium supplemented with $10 \%$ fetal bovine serum (FBS), 100 $\mathrm{U} / \mathrm{ml}$ penicillin and $100 \mathrm{mg} / \mathrm{ml}$ streptomycin at $37^{\circ} \mathrm{C}$ in a water-saturated atmosphere with $5 \% \mathrm{CO} 2 / 95 \%$ air. The cell line was verified in writing as being ovarian in origin and no mycoplasma contamination.

\section{Animal experiments}

Animal experiments were approved by the Animal Research Committee of Hirosaki University and all animals were cared for and handled in accordance with the Rules for Animal Experimentation of Hirosaki University and animal practices as defined by national and local bodies governing animal welfare (Guide for the Care and Use of Laboratory Animals published by the National Institutes of Health). Eight-week-old female F344 rnu/ rnu rats weighing 180 to $200 \mathrm{~g}$ and eight-week-old female $\mathrm{BALB} / \mathrm{c}$ nu/nu mice weighing 20 to $25 \mathrm{~g}$ (CLEA Japan, Tokyo, Japan) were used in this study. All animals were group housed in plastic cages with stainless-steel grid tops in an air-conditioned and $12 \mathrm{~h}$-light-dark-cyclemaintained room and fed with water and food ad libitum in the Institute for Animal Experiments of Hirosaki University.

\section{Rat model of peritoneal carcinomatosis}

DISS cells were grafted into the rat abdomen at a concentration of 2 X106/ml. On day 10 after grafting, tumor formation in the omentum and peritoneal dissemination were noted (Figure 1). In order to examined the effectiveness of PDT at treating peritoneal dissemination, 1) rats were divided into a control group $(n=5)$ receiving light irradiation alone and a methyl-ALA-PDT group $(n=5)$ that received methyl-ALA intraperitoneally followed by PDT. The survival time of the two groups was compared. In order to examine the effectiveness of DS, PDT, and CA on peritoneal dissemination, 2) rats were divided into a control group that received light irradiation alone, a DS group that received light irradiation following DS, a DS+methyl-ALA-PDT group that received DS after administration of methyl-ALA followed by PDT, and a DS+methyl-ALA-PDT+CA group that received DS after administration of methyl-ALA followed by PDT and daily administration of CA. The survival time of the four groups was compared. There were 5 rats in each group.

Administration of 5-aminolevulinic acid methyl ester hydrochloride (methyl-ALA)

Methyl-ALA was purchased from Cosmo Bio International (Tokyo, Japan). Methyl-ALA was promptly dissolved in PBS to reach a concentration of $10 \mathrm{mg} /$ $\mathrm{ml}$. The PDT group was intraperitoneally administered $250 \mathrm{mg} / \mathrm{kg}$ of methyl-ALA $3 \mathrm{hrs}$ prior to light irradiation, and the group receiving light irradiation alone was intraperitoneally administered only PBS (Wakui et al., 2010).

\section{Light irradiation}

Three hrs after administration of methyl-ALA, rats underwent a laparotomy. Rats were immobilized on an operating table and then irradiated with light from a halogen lamp ( $150 \mathrm{~W}$, wavelength: $600 \mathrm{~nm})$ positioned at a height of $10 \mathrm{~cm}$. Light irradiation lasted $10 \mathrm{~min}$. The dose of light energy was $90 \mathrm{~J} / \mathrm{cm}^{2}$ (Figure 1).

Table 1. Amount of Protoporphyrin IX after Administering a Methyl Ester of 5-Aminolevulinic Acid

\begin{tabular}{lc}
\hline Organs & Amount of protoporphyrin IX (mg/dl) \\
\hline Peritoneal foci of cancer & $98.6 \pm 25.5$ \\
Muscle & $5.1 \pm 1.2$ \\
Kidney & $5.5 \pm 2.5$ \\
Skin & Not detectable \\
Liver & Not detectable \\
Large intestine & Not detectable \\
Small intestine & Not detectable \\
\hline
\end{tabular}




\section{Debulking surgery and sham operation}

Laparotomy was performed under pentobarbital anesthesia and sterile condition. The omentum was reached via a $4-\mathrm{cm}$ midline incision in the abdomen and subsequently extirpated by ligating gastroepiploic vessels and hilum of the spleen (Yokoyama et al., 2012). Sham operation was done by a similar incision. After hemostasis, the abdominal wall was closed in two layers using $3 / 0$ polyglactin (Vicryl®, Ethicon, Tokyo, Japan). No animal died as a result of the operative procedure.

\section{Clofibric acid (CA) administration}

DS+Methyl-ALA-PDT+CA group was given 9,000 ppm CA (Sigma-Aldrich, St Louis, MO) in the diet everyday after DS until the end of the study.

Accumulation of the methyl ester of aminolevulinic acid in tumor tissue and normal tissue and tissue damage

Methyl-ALA is a photosensitizer precursor with an affinity for cancer. Methyl-ALA is converted into protoporphyrin via the heme biosynthesis pathway in cells, and protoporphyrin produces a photochemical action

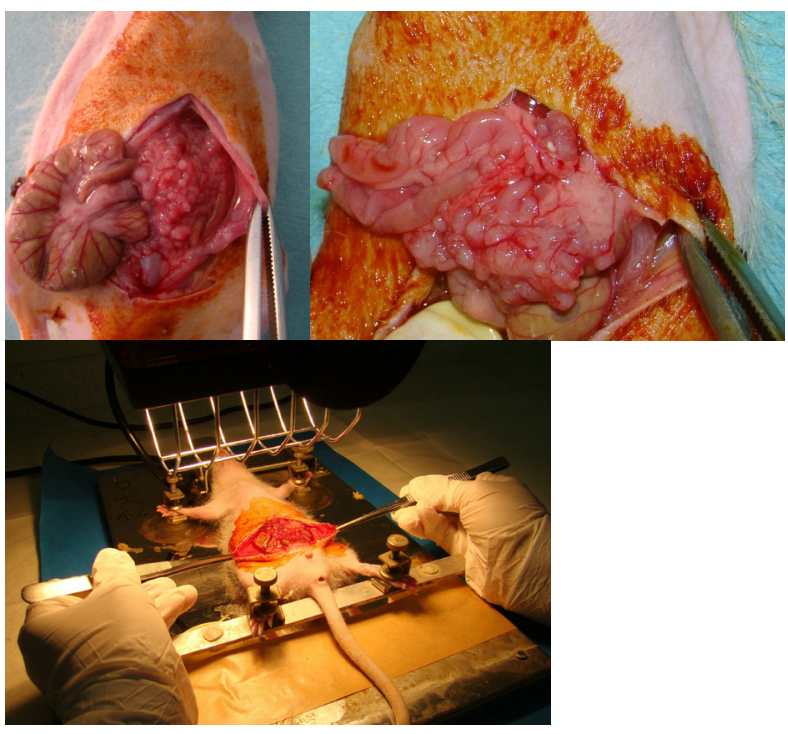

Figure 1. Rat Model of Peritoneal Carcinomatosis and Light Irradiation

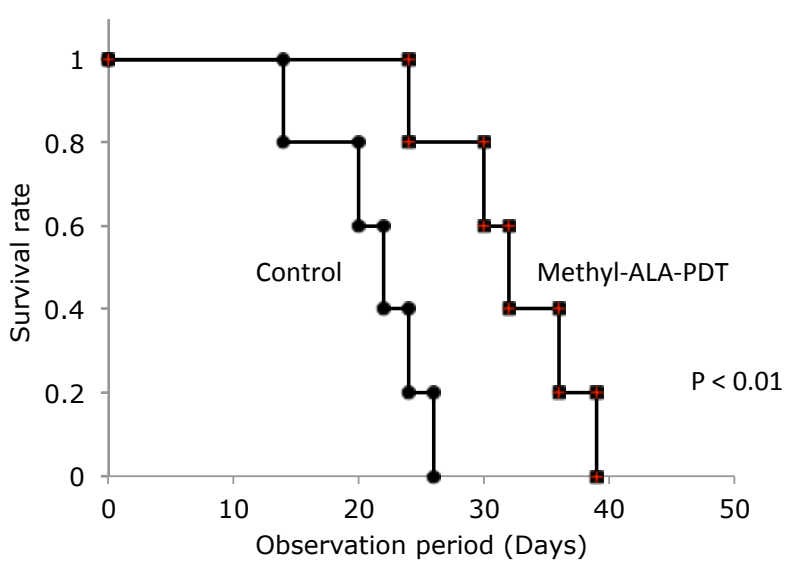

Figure 2. The Effectiveness of PDT in a Rat Model of Peritoneal Carcinomatosis. Rats that received PDT with methyl-ALA had a significantly longer survival time $(\mathrm{P}<0.01)$ when activated by light. DISS cells at a concentration of $1 \times 10^{5} / \mathrm{ml}$ were intraperitoneally grafted into 5 nude mice. Peritoneal carcinomatosis developed, and in the second week after the graft methyl-ALA was administered intraperitoneally at a dose of $250 \mathrm{mg} / \mathrm{kg}$ body. Three hrs after administration, tumors, the skin, muscle, and major organs in the abdomen, i.e. the liver, the kidneys, and the intestines, were removed and assayed for protoporphyrin using HPLC (Wakui et al., 2010). Five mice with peritoneal carcinomatosis were intraperitoneally administered methyl-ALA at a dose of $250 \mathrm{mg} / \mathrm{kg}$ body. Three hrs later, the abdominal cavity was irradiated with light from a halogen lamp for $10 \mathrm{~min}$. Twenty-four hrs later, tumors, the skin, muscle, and major organs, i.e. the liver, the kidneys, and the intestines were removed. Tissues were fixation with formalin and pathologically examined for necrotic changes.

\section{Statistical analysis}

The survival curves were calculated by the KaplanMeier method, and the statistical significance of differences in the cumulative survival curves between the groups was evaluated by logrank test. A result was deemed significant at $\mathrm{P}<0.05$.

\section{Results}

The effectiveness of PDT in a rat model of peritoneal carcinomatosis

Rats that received PDT with methyl-ALA had a median survival time of $33 \mathrm{~d}$. Rats that received PDT alone had a median survival time of $22 \mathrm{~d}$, so rats that received PDT with methyl-ALA had a significantly longer survival time (Figure 2, $\mathrm{P}<0.01$ ).

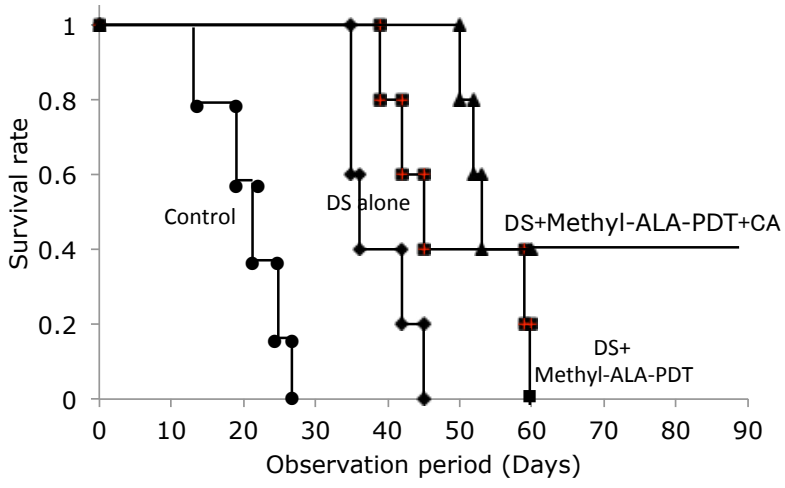

Figure 3. Effectiveness of a Concurrent Therapy with PDT and CA following DS in a rat model of peritoneal carcinomatosis. Rats that received DS alone had a significantly longer survival time compared to the controls $(\mathrm{P}<0.05)$. Rats that received DS+methyl-ALAPDT tended to have a longer survival time compared to the rats that received $\mathrm{DS}$ alone $(\mathrm{P}=0.08)$. Rats that received DS+methyl-ALA-PDT+CA had a significantly longer survival time compared to the rats that received DS alone $(\mathrm{P}<0.005)$. Note that 3 of the 5 rats that received DS+methyl-ALA-PDT+CA died, but the remaining 2 rats survived for $90 \mathrm{~d}$ 


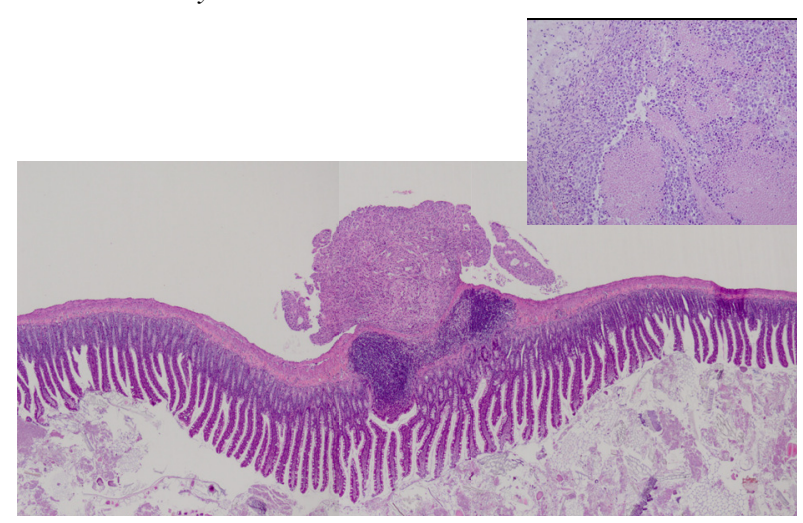

Figure 4. Microphotograph of Disseminated Lesion and the Intestine After Methyl-ALA-PDT. While necrotic changes occurred in tumors (extended image), abnormalities in the intestinal wall were not noted

Effectiveness of a concurrent therapy with PDT and CA following DS in a rat model of peritoneal carcinomatosis

Rats were observed for $90 \mathrm{~d}$. Rats that received DS alone had a mean survival time of $35.5 \mathrm{~d}$. Rats that received DS+methyl-ALA-PDT had a mean survival time of $46.3 \mathrm{~d}$, and rats that received DS+methyl-ALAPDT+CA had a mean survival time of $52.5 \mathrm{~d}$ ( 3 of the 5 rats in this group died, but the remaining 2 rats survived for $90 \mathrm{~d}$ ). Rats that received DS alone had a significantly longer survival time compared to the controls (Figure 3, $\mathrm{P}<0.05)$. Rats that received DS+methyl-ALA-PDT tended to have a longer survival time compared to the rats that received DS alone (Figure 3, $\mathrm{P}=0.08$ ). In addition, rats that received DS+methyl-ALA-PDT+CA had a significantly longer survival time compared to the rats that received DS alone (Figure 3, $\mathrm{P}<0.005$ ). There were no deaths due to surgery.

\section{Protoporphyrin levels in organs}

The protoporphyrin level in tumors in the abdomen was $98.6 \pm 25.5 \mu \mathrm{g} / \mathrm{dl}$ (Table 1). Low levels of protoporphyrin were detected in muscle and the kidneys, and levels in skin, the liver, the large intestine, and the small intestine were below the detection sensitivity of the assay (Table 1). A look at the relationship between disseminated lesions and the intestines in terms of pathology indicated that necrotic changes occurred in tumors, but abnormalities in the intestinal wall were not noted (Figure 4).

\section{Discussion}

The current results indicated that concurrent therapy consisting of PDT with methyl-ALA and CA is effective at treating peritoneal carcinomatosis resulting from ovarian cancer. Protoporphyrin, a metabolite of methyl-ALA, triggers a photochemical action when activated by light. Protoporphyrin was not detected in major organs in the abdomen and was highly concentrated only in tumors. Thus, PDT with methyl-ALA does not appear to cause organ damage.

The antitumor action of PDT with methyl-ALA is a result of the induction of apoptosis and the inhibition of angiogenesis (Wakui et al., 2010). Protoporphyrin levels in cells indicated this antitumor action (Wakui et al., 2010). CA induces carbonyl reductase, which inactivates prostaglandin in a tumor. CA also inhibits COX-2-induced angiogenesis and it induces apoptosis, which explains its antitumor action (Yokoyama et al., 2007). In the current study, these 2 mechanisms presumably led to the control of peritoneal dissemination.

PDT is clinically used to treat early lung cancer, esophageal cancer, gastric cancer, cervical cancer, and superficial skin malignancies (Casson,2009; Ikeda et al., 2011; Spinelli et al., 1992; Choi et al., 2014; Lucena et al., 2015). The drug Photofrin is used in PDT, but it has several flaws. Photofrin is expensive, and patients must spend several hours in the dark while the drug is being eliminated from normal cells in order to avoid photosensitivity. Thus, what is needed is a drug that is less expensive, that reacts at light at wavelengths that penetrate tissue, that is quickly eliminated from normal tissue, that causes little photosensitivity, and that is highly cytotoxic. In the current study, PDT with methyl-ALA involved a light dose of 90 $\mathrm{J} / \mathrm{cm} 2$ from a halogen lamp. There was no tissue damage, and the wavelength of light from the halogen lamp was similar to that of light from a surgical lamp. Thus, PDT with methyl-ALA is quite suited to clinical use.

Basic research on PDT is progressing in the area of uterine cervical cancer and the efficacy of PDT on this disease has been established (Liu et al., 2013; Wei et al., 2013). Hypocrellin B is a photosensitizer. Recent study has reported that a nanoparticle drug delivery system causes hypocrellin B to be specifically taken up by cancer cells and that PDT has increased effectiveness at treating peritoneal carcinomatosis resulting from ovarian cancer (Li et al., 2015). Erlotinib is a drug that targets epidermal growth factor receptors, and administering that drug beforehand is reported to increase the effectiveness of PDT (Gallagher-Colombo et al., 2015). Axitinib is a VEGF receptor tyrosine kinase inhibitor, and administering that drug beforehand is reported to improve the antitumor action of PDT (Weiss et al., 2015). Approaches have been devised to that PDT can be used to treat peritoneal carcinomatosis and drug-resistant cancer. Research on PDT is progressing particularly in the area of ovarian cancer treatment. Rizvi et al. (2010) reported that a combination of carboplatin and PDT had a synergistic effect when treating ovarian cancer micrometastases. A combination of an immunotherapy that blocks EGFR and PDT is reported to have a synergistic effect when treating ovarian cancer (del Carmen et al., 2005). Similarly, the current study found that a combination of PDT and CA after DS was effective at controlling peritoneal dissemination of ovarian cancer.

A light dose of $90 \mathrm{~J} / \mathrm{cm} 2$ was used in the current study. Whether this light dose or an approach like nanoparticle drug delivery is appropriate at increasing the uptake of methyl-ALA by a tumor must be assessed. Those findings will facilitate the clinical use of PDT to treat peritoneal dissemination. Further studies such as toxicity studies should be conducted in the future. 


\section{Acknowledgements}

This study was supported by a Grant-in-Aid for Cancer Research from the Ministry of Education, Culture, Sports, Science and Technology (Tokyo, Japan) (no. 20591935 to Dr Y. Yokoyama).

\section{References}

Akeson M, Zetterqvist BM, Dahllöf K, et al (2008). Effect of adjuvant paclitaxel and carboplatin for advanced stage epithelial ovarian cancer: a population-based cohortstudy of all patients in western Sweden with long-term follow-up. Acta ObstetGynecol Scand, 87, 1343-52.

Casson AG (2009). Photofrin PDT for early stage esophageal cancer: a new standard of care? Photodiagnosis Photodyn Ther, 6, 155-6.

Choi MC, Lee C, Kim SJ (2014). Efficacy and safety of photodynamic therapy for cervical intraepithelial neoplasia: a systemic review. Photodiagnosis Photodyn Ther, 11, 479-80.

del Carmen MG, Rizvi I, Chang Y, et al (2005). Synergism of epidermal growth factor receptor-targeted immunotherapy with photodynamic treatment of ovarian cancer in vivo. $J$ Natl Cancer Inst, 97, 1516-24.

Gallagher-Colombo SM, Miller J, Cengel KA, et al (2015). Erlotinib Pretreatment Improves Photodynamic Therapy of Non-Small Cell Lung Carcinoma Xenografts via multiple Mechanisms. Cancer Res, 75, 3118-26.

Ikeda N, Usuda J, Kato H, et al (2011). New aspects of photodynamic therapy for central type early stage lung cancer. Lasers Surg Med, 43, 749-54.

Kroep JR, Nortier JW (2012). The role of bevacizumab in advanced epithelial ovarian cancer. Curr Pharm Des, 18, 3775-83.

Lee CK, Lord S, Grunewald T, et al (2015). Impact of secondary cytoreductive surgery on survival in patients with platinum sensitive recurrent ovarian cancer: analysis of the CALYPSO trial. Gynecol Oncol, 136, 18-24.

Lucena SR, Salazar N, Gracia-Cazaña T, et al (2015). Combined treatments with photodynamic therapy for non-melanoma skin cancer. Int J Mol Sci, 16, 25912-33.

Li Z, Sun L, Lu Z, et al (2015). Enhanced effect of photodynamic therapy in ovarian cancer using a nanoparticle drug delivery system. Int J Oncol, 47, 1070-6.

Liu AH, Sun X, Wei XQ, et al (2013). Efficacy of multiple lowdose photodynamic TMPYP4 therapy on cervical cancer tumour growth in nude mice. Asian Pac J Cancer Prev, 14, 5371-4

Peng Q, Warloe T, Moan J, et al (2001). Antitumor effect of 5-aminolevulinic acid-mediated photodynamic therapy can be enhanced by the use of a low dose of photofrin in human tumor xenografts. Cancer Res, 61, 5824-32.

Rizvi I, Celli JP, Evans CL, et al (2010). Synergistic enhancement of carboplatin efficacy with photodynamic therapy in a three-dimensional model for micrometastatic ovarian cancer. Cancer Res, 70, 9319-28.

Siegel R, Naishadham D, Jemal A (2013). Cancer statistics, 2013. CA Cancer J Clin, 63, 11-30.

Spinelli P, Dal Fante M, Mancini A (1992). Current role of laser and photodynamic therapy in gastrointestinal tumors and analysis of a 10-year experience. Semin Surg Oncol, 8, 204-13.

Wakui M, Yokoyama Y, Wang H, et al (2010). Efficacy of a methyl ester of 5-aminolevulinic acid in photodynamic therapy for ovarian cancers. J Cancer Res Clin Oncol, 136,
1143-50.

Wang C, Chen X, Wu J, et al (2013). Low-dose arsenic trioxide enhances 5-aminolevulinic acid-induced PpIX accumulation and efficacy of photodynamic therapy in human glioma. $J$ Photochem Photobiol B, 127, 61-7.

Wei XQ, Ma HQ, Liu AH, et al (2013). Synergistic anticancer activity of 5-aminolevulinic acid photodynamic therapy in combination with low-dose cisplatin on Hela cells. Asian Pac J Cancer Prev, 14, 3023-8.

Weiss A, Bonvin D, Berndsen RH, et al (2015). Angiostatic treatment prior to chemo- or photodynamic therapy improves anti-tumor efficacy. Sci Rep, 5, 8990.

Yokoyama Y, Xin B, Shigeto T, et al (2007). Clofibric acid, a peroxisome proliferator activated receptor a ligand, inhibits growth of human ovarian cancer. Mol Cancer Ther, 6 , 1379-86.

Yokoyama Y, Hirakawa H, Wang H, et al (2012). Is omentectomy mandatory in the operation for ovarian cancer? Eur J Obstet Gynecol Reprod Biol, 164, 89-92.

Yu J, Cao XF, Zheng Y, et al (2014). Anti-VEGF Therapy with Bevacizumab--limited cardiovascular toxicity. Asian Pac J Cancer Prev, 15, 10769-72. 